\title{
Vibration-based delamination diagnosis and modelling for composite laminate plates
}

\author{
D.Garcia $^{\mathrm{a}}$, R.Palazzetti ${ }^{\mathrm{b}, *}$, I.Trendafilova $^{\mathrm{a}}$, C.Fiorini $^{\mathrm{c}}$, A.Zucchelli $^{1}$ \\ ${ }^{a}$ University of Strathclyde, MAE department, 75 Montrose Street, G1 1XJ, Glasgow, UK \\ ${ }^{b}$ University of Strathclyde, DMEM department, 75 Montrose Street, G1 1XJ, Glasgow, UK \\ ${ }^{c}$ University of Bologna, Department of Industrial Engineering, viale del Risorgimento 2, 40136 \\ Bologna, Italy
}

\begin{abstract}
This study suggests a methodology for damage diagnosis in structures, which is based on a multivariate statistical procedure and uses the measured time domain structural vibration response. In this paper the methodology is developed for the purposes of delamination assessment in free vibrating composite laminate plates. It applies singular spectrum analysis to the measured strains in order to decompose and compress the information contained in these responses into a smaller number of independent variables. The method works on the principle of clusterization between healthy and delaminated plates with different delamination sizes and locations. The developed methodology is then applied and verified on numerically simulated and experimentally obtained results for composite laminate plates. The results demonstrate a good capability for clusterization between different delamination scenarios in composite laminate plates.
\end{abstract}

Keywords: delamination detection, VSHM, composite plates, SSA

\section{Introduction}

Composite laminates are composite materials made by stacking composite layers in a sequence depending on the final usage of the laminate. Delamina-

\footnotetext{
* Principal corresponding author

Email addresses: david.garcia@strath.ac.uk (D.Garcia),

roberto.palazzetti@strath.ac.uk (R.Palazzetti), irina.trendafilova@strath.ac.uk (I.Trendafilova), cosimo.fiorini@gmail.it (C.Fiorini), a.zucchelli@unibo.it (A.Zucchelli)
} 
tion is the most common failure mode for laminates, and it can be due to different reasons including impacts, fatigue loading and excessive strain [1]. Delamination is a particularly dangerous failure mode, because it takes place and grows under the surface without being visible from the outside, causing matrix breakage and leading to changes in physical properties of the whole laminate such as reduction in stiffness and strength.

This study suggests the use of the vibration response of a laminate to monitor its health. Structural Health Monitoring (SHM) techniques use non-destructive procedures to derive conclusions for the health and the condition of a structure. Vibration-based SHM (VSHM) methods present a very attractive possibility since they are global non-destructive methods and do not require any a priori information for the damage location, type or size. There exists a number of VSHM methods that have been developed, some of which are based on the modal parameters of a structure, including its natural frequencies [2] and/or mode shapes [3], others use its Frequency Response Functions (FRFs) and/or its time or frequency domain vibration response [4]. In general VSHM methods can be divided into two big categories: model-based and non-model based methods. The modelbased methods are generally based on the comparison between the measured and the modelled vibration response. Eventually the damage identification is made minimizing the difference between the measured and the modelled structural vibration responses. Different distances can be used to measure this difference and different updating techniques have been used to bring the model as close as possible to the measured response and thus find the parameters that characterize the delamination like e.g location, extent or more precise dimensions or coordinates [5]. Composite laminates and structures made of composites in general demonstrate nonlinear dynamic behaviour in the sense of time series nonlinearity and in the sense of nonlinearities with respect to different parameters. In such a sense, modelling the vibratory response of such structures is difficult especially if precise prediction is required since the traditional modelling techniques cannot be used. There are some studies that target model-based VSHM methods as applied to composite structures but it should be stressed that these are developed for specific applications in the sense of material and structures and they are generally not applicable to other cases. FEM has been used by several authors for the purposes of modelling the response of structures made of laminates, especially beams and a few of them are focused on plates [6-13]. A number of works can be found dealing with modelling the vibratory response of delaminated composite laminate structures [6-10]. Even though some methods can relatively precisely model the presence of delamination, little research is available on the topic of delamination 
detection, which involves dynamic modelling of laminate structures [11--13]. Thus it should be noted that VSHM methods developed for structures made of composites and or composite laminates tend to be non-model based [14]. The non-model based VSHM methods do not use any model and they do not assume any linearity for the vibratory behavior of a structure. They are solely based on the analysis of its vibration response in terms of e.g. natural frequencies, time series analysis and/or pure data analysis procedures. A previous paper by some of the authors of this work [15] treats the first six modal frequencies of composite beams and their changes due to the presence of delamination. Authors showed that to a certain extent natural frequencies can be successfully used to investigate the presence, the location and the dimensions of the delamination in a composite beam. But the problem with using the natural frequencies for structures made of composites can be a rather difficult job because of their well-expressed nonlinear vibratory behaviour which results in e.g. double or very close natural frequencies which cannot be found using the spectrum of the vibration response. Moreover it is generally the higher frequencies that contain information about the presence and the parameters of localized damage which are still more difficult to identify from experiment.

Thus a lot of authors suggest the application of purely data driven statistical and data analysis methods for the purposes of extracting information for the delamination from the measured vibration response. In [16] the application of signal cross correlation is considered for similar purposes of delamination detection and localization. The data driven methods are targeting different characteristics of the signals recorded, they are in principle data analysis methods and thus can be applied for any signals, including deterministic and random ones. There are several papers that suggest the use of multivariate data analysis methods, specifically principal component analysis (PCA) for the purposes of VSHM [17-20] because they reveal correlation between the measured vibration characteristics. PCA has been applied for delamination detection and classification with results that prove its potential for damage detection [17]. Tibaduiza et al. [18] present PCA as a tool for damage classification with the extension of self-organizing maps. A.M. Yan et al. [19] detail in two papers the use of PCA as a linear tool for correlating the vibration characteristics and environmental conditions in order to detect damage in structures. Subsequently, they present an extension for non-linear cases [20] where PCA is applied firstly to cluster the data and secondly locally for each set of data. These methodologies apply PCA on certain values or characteristics of the vibration responses of the structure like e.g. amplitude, frequency and impedance. However, applied in this manner PCA will only be able to uncover in- 
formation contained at these particular time or frequency data points. In contrast the methodology proposed here is able to uncover information and particularly rotational patterns at any frequency throughout the whole frequency range of the measured signal. In turn this results in a very precise reconstruction of the original signal.

It should be noted that PCA possesses several favourable properties as applied for damage/delamination assessment purposes. It first reduces the dimension of the measured data, in our case this reduces the dimension of the measured vibration signals. Furthermore it possesses clustering or categorization properties in the sense that the new components tend to make clusters corresponding to the different categories of data: in our case signals from intact structures and those from structures with different sizes and locations of delamination. This is achieved by reducing the distance between data vectors from the same category while in the same time increasing the distance between data vectors from different categories. It also preserves the information contained in the original signals in terms of variability.

The method suggested here is based on PCA which is a data analysis method and thus it uses solely the measured vibration response in order to extract information about the structural vibratory behavior and its health. Singular Spectrum Analysis (SSA) is a natural extension of PCA which is specifically developed for time series [21] and it possesses properties which are beneficial and make it appropriate for the analysis of dynamic/vibratory behavior. SSA is able to uncover the rotational patterns of the measured response and it is especially appropriate for non-linear and non-harmonic rotations and periodicities since in contrast to spectrum analysis it is capable to uncover periodicities at any frequency. This is why it was initially developed for applications in climatology, meteorology and social sciences [22], but the first development of SSA for structural vibration analysis and for the purposes of VSHM belongs to some of the authors of this study [21]. In addition SSA as an alternative of PCA features properties that are favorable for damage assessment and for the analysis of data from different categories. It first reduces the dimension of the measured data, in our case this significantly reduces the dimension of the measured vibration signals. Furthermore it possesses clustering or categorization properties in the sense that the new components tend to make clusters corresponding to the different categories of data in our case signals from intact structures and those from structures with different sizes and locations of delamination. This is achieved by reducing the distance between data vectors from the same category while in the same time increasing the distance between data vectors from different categories. It also preserves the information contained 
in the original signals in terms of variability.

There is only one other application of SSA that the authors are aware of, for the purposes of bridges monitoring [23]. SSA is principally a data analysis method which can be applied in the time and/or the frequency domain. This study looks at the application of SSA in the frequency domain. The frequency domain strain signals measured on a structure are subjected to SSA in order to extract useful information and features that can be used for delamination assessment. Only few of the first principal components containing most of variance of the initial signals are used to approximate each vibration signal.

The rest of the paper is organized as follows. The next paragraph introduces the delamination assessment methodology. $\S 3$ is dedicated to the case study of a laminate plate: its free vibratory behaviour without delamination and in the presence of several delamination scenarios is simulated by using FE modelling. An experimental study is also performed to obtain the free decay responses of a laminate plate with the same dimensions and characteristics as the simulated one under the same delamination scenarios (including the healthy plate). $\S 4$ presents the results from the application of the methodology for delamination diagnosis, which are further discussed from view point of their application to the experimentally and the FE simulated vibratory responses. The results validate the delamination assessment capabilities of the methodology. The paper finishes with some conclusions regarding the methodology and this specific application.

\section{The SSA-based methodology as applied to composite laminate plates}

In this paragraph the SSA-based methodology is applied for composite laminate plates.

The vibration characteristic used in this present study is the in-plain strain of the outer layer of the considered specimens. First the discrete vibratory responses are represented as vectors. Then, the vector signals are transformed into the frequency domain where data are ordered and the information is distributed and gathered at particular frequency points. Furthermore, the lagged frequency vectors are decomposed into a certain number of Principal Components (PCs), containing the variance of the original data, whereas the first PCs contain most of this variance. The decomposed components are eventually used to build delamination recognition features using the capabilities of PCA of separating data from different categories. A detail of the methodology is here described [21]:

- Data collection: the signals measured from the composite laminate plates, in this particular case the dynamic strain, for both FEM and experimen- 
tal test, are arranged into $\mathrm{N}$-dimensional vectors in the following form $x=$ $\left(x_{1}, x_{2}, \ldots, x_{N}\right)$. Since the strains have different magnitudes, the values are first standardized. The time domain vectors are then transformed into frequency domain signal vectors as $z=\left(z_{1}, z_{2}, \ldots, z_{N / 2}\right)$.

- Embedding: given a window with length $W\left(1<W \leq \frac{N}{2}\right)$, the $W$ - frequency lagged vectors arranged in columns are used to define the embedding matrix $\widetilde{Z}$, which is a Hankel matrix. These vectors are padded with zeros to keep the same vector length. The embedding matrix $\widetilde{Z}$ is the representation of the system in a succession of overlapping vectors of the frequency series by $W$-points. All the information about the dynamics of the vibratory response of the composite laminate plates is contained into the embedding matrix;

- Decomposition: the principal directions of the system, called Empirical Orthogonal Functions (EOFs) are calculated by the eigen-decomposition of the covariance matrix of $\widetilde{Z}$. The eigen-decomposition yields $k$-eigenvalues and $k$-eigenvectors, which define the orthonormal basis of the decomposition of $\widetilde{Z}$;

- Reconstruction: The EOFs represent the data as a decomposition of the orthogonal basis functions with a certain percentage of the variance of the original signal corresponding to each EOF. The data contained in the matrix $\widetilde{Z}$ is projected onto the subspace $\mathscr{L}^{k}$ built by the EOFs where the corresponding PCs are obtained [24]. The oscillatory responses of the system can be reconstructed using a certain number of the new variables, called reconstructive components (RCs). Each one of the RCs contains a certain percentage of variance of the initial variables provided by the EOFs;

- Projection: Each RC is a separated signal reconstruction of the reference signal (undamaged signal) based on the mean and variance contained in its corresponding PC. The RCs are ordered from largest to lowest content of variance. Therefore, $\mathrm{RC} 1$ and $\mathrm{RC} 2$ are associated with the highest variance content (highest singular value) and they are responsible for the greatest variation of the vibration features. The data signals from damage scenarios (Observation data, $\mathbf{O}$ ) are then projected according to Eq. 4 onto the RCs. This projection results in a set of scalar points namely as $\mathbf{T}$. In this particular case the projection is considered as two dimensional space for visualization proposes. 


$$
T=\langle O, R\rangle
$$

- Clusterization: The points projected on the new subspace (T) are used as damage features for delamination assessment. The data projected will distribute and cluster depending on the similarities of mean and variance contained in this new subspace. As delamination affects the mass, stiffness and global/local structural behaviour, it also makes changes in the rotational patterns uncover in their vibration response. These will modify or introduce new oscillatory components, harmonics or non-harmonics, which will be present in the vibratory response. Therefore, the points projected in the new subspace will reduce their distances with points from the same category and increase the distance from points of different categories. As effect, the data-points create clusters which differ between different categories.

The methodology is able to compress the relevant information contained in the vibratory signals and thus it is able to detect most of the changes due to delamination. This information is projected onto a new space where the data is better observable and can be better analysed. The obtained RCs in step 4 are further used as delamination features in this study. For the purposes of visualization only the first two RCs are used, which contain the majority of the variance of the original data. As a result of the categorization capabilities of the developed procedure, the RCs form several different delamination categories are well separated, which make possible the recognition between healthy and delaminated plates and also among the different delamination scenarios.

In a previous paper [21] the authors explore the application of the suggested method for a 2 DoF system with linear stiffnesses and a nonlinear coupling stiffness. Damage was simulated by reducing some of the system stiffnesses and principally the same method was applied for the purposes of diagnosis of the different stiffness reductions/damages. The results show that the suggested method is capable to identify linear as well as nonlinear stiffness reductions. The suggested method actually performs better for linear stiffness variations as compared to the case of nonlinear stiffness reductions. Rather small changes in the linear stiffness (less than 10 percent) are diagnosed correctly while the same changes in the nonlinear stiffness might give confusing results. In such a sense it can be said that the suggested methodology is affected by the nonlinear character of the damage. But it should be stressed that it works for linear as well as for nonlinear damage types. 


\section{Simulations and experiments}

In this chapter the methodology described in $\$ 2$ is applied to a case study of composite laminate plates.

\subsection{Finite element modelling}

Solid 186 elements from ANSYS library have been used to model 10 layers, $150 \mathrm{~mm}$ side, squared laminate plates. The material properties are as follow: $E_{1}$ $=E_{2}=59 \mathrm{GPa}, E_{3}=9 \mathrm{GPa}, G_{12}=G_{13}=G_{23}=7.17 \mathrm{GPa}, v_{12}=v_{13}=v_{23}=0.3$ and $\rho=1500 \mathrm{~kg} / \mathrm{m}^{3}$.

Plates were modelled using 225 elements per layer. Four different plates were modelled: a non-delaminated configuration, denoted by the character " $\mathrm{H}$ ", and three delaminated configurations, labelled with D1, D2 and D3 (see Figure 1):

- in D1 configuration, delamination is placed in the middle of the plate, between the $5^{\text {th }}$ and $6^{\text {th }}$ layers;

- in D2 configuration, delamination is again in the middle of the plate, placed between the $8^{\text {th }}$ and $9^{\text {th }}$ layers;

- in D3 configuration, delamination is moved closer to a corner, having it centre at $3 / 4$ of each side, between the $5^{\text {th }}$ and $6^{\text {th }}$ layers.

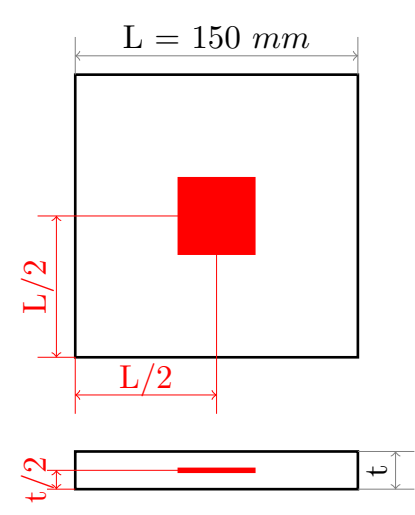

(a) D1

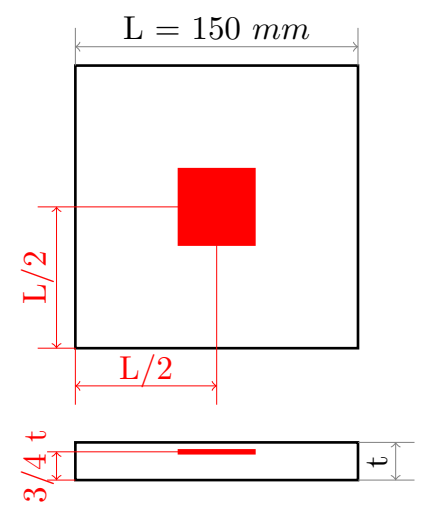

(b) D2

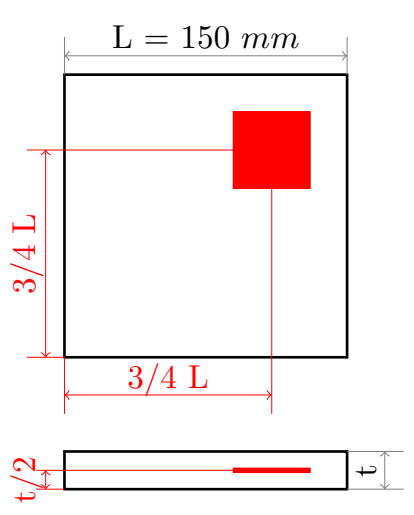

(c) D3

Figure 1: Delamination configurations

For all the configurations, delamination size was $40 \mathrm{~mm} \times 40 \mathrm{~mm}$, and has been simulated as an actual separation, of $0.05 \mathrm{~mm}$ between two layers of the plate. 
Delaminated specimens are modelled following the regional approach proposed by Mujumdar et al. [10] and the mesh was further refined in the delaminated region. The two surfaces identifying the delamination are modelled using TARGE170 (upper surface) and CONTA174 (lower surface, the target) elements, which break the contact in the delaminated region, allowing friction and impact between the two interfaces when the plate vibrates. The free vibration responses where obtained by applying a short/burst impulse in a certain point on the plate and the free decay response was measured after the application of the pulse load. Inplane strain was extracted in correspondence of one of those points where the strain gauges were placed in the tested samples. In order to simulate a real experiment, a $10 d B$ SNR (signal to noise ratio) was added to each signal realization. This noise contamination is used to introduce small alteration in the vibratory responses. Signals are recorded for $1 \mathrm{~s}$ and sampled at $4098 \mathrm{~Hz}$.

The natural frequencies of the simulated delaminated plates where obtained by performing transient analysis, chosen in order to include the non-linear effects due to contact breakage, delamination opening and closing (delamination breathing), contact/impacts and friction between the delaminated layers during vibration. The frequency spectrum of the vibratory response was calculated from the time-domain response via the Fourier transformation: the peaks of the resulting spectrum indicate which frequencies are resonant. CFRP composite materials have small damping and then resonant frequencies are approximately equal to the natural frequencies of the system. Therefore, the frequencies with larger amplitude can be considered to be the natural frequencies of vibration.

\subsection{Experiments}

Specimens of the same configuration as those numerically simulated have been manufactured and tested to validate the method suggested. Delamination was introduced by laying down a $40 \mathrm{~mm}$ x $40 \mathrm{~mm}, 15 \mu \mathrm{m}$ thick Teflon ply during lay up (see Figure 2(a); specimens have been cantilevered on one edge (see Figure 2(b) and a bump test has been performed using a steel hammer, while two strain gauges applied on the upper surface of the plates (see Figure 2(c) recorded the strain and thus the vibration of the plates. The bump test was manually performed, and the beam's deformations were kept within the small displacement field.

The external in-plane strain was recorded by strain gauges powered with a P-3550 Strain Indicator; an analogue-to-digital acquisition device digitalized the signal which was then acquired on a personal computer by a Labview executable program. Sampling frequency and acquisition time were $1.25 \mathrm{kHz}$ and $1.638 \mathrm{~s}$ respectively. Each specimen was tested 5 times and the results are given in terms 


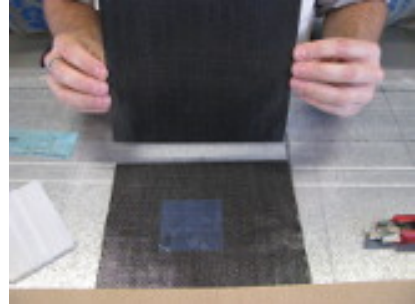

(a) Teflon to induce delamination

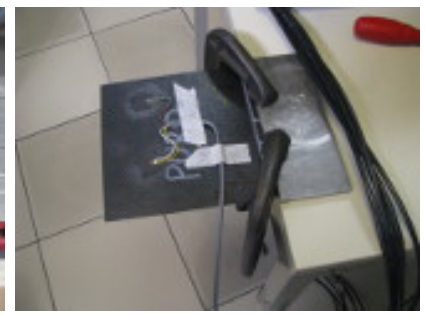

(b) Experiments set up

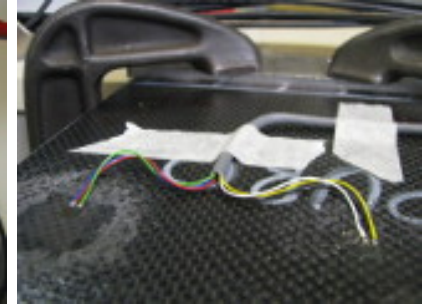

(c) Strain gauges

Figure 2: Experimental set-up

of average of all the tests.

Manufactured plates were subjected to a bump test and the vibratory response was recorded by measuring the external in-plan stain. The signals have then been processed in the same way the numerically obtained ones had.

\subsection{FEM modeling and validation}

To validate the numerical model from $\S 3.1$, the first 5 natural frequencies of vibration of the numerically simulated plate were calculated and compared to those obtained using the experimentally obtained ones. Figure 3 presents the natural frequencies of vibration of undamaged and delaminated configurations, showing very good agreement between the numerical and the experimental results. The proposed model is accurate enough to simulate the dynamic behavior of a composite laminate plate, with and without delamination.

Furthermore the authors would like to point out the presence of double/very close modes around at $500 \mathrm{~Hz}$ (see Figures 4(b) 4(c) 5(b) 5(c)), which is a phenomenon typical for composite structures and for laminates. This can be observed in our simulation as well as in the experimental results.

\section{Delamination assessment capabilities of the suggested methodology}

In this paragraph the methodology as introduced in $\$ 2$ is applied to the numerical simulation results first (\$3.1) and subsequently to the experimentally ones $\$ 3.2$. 


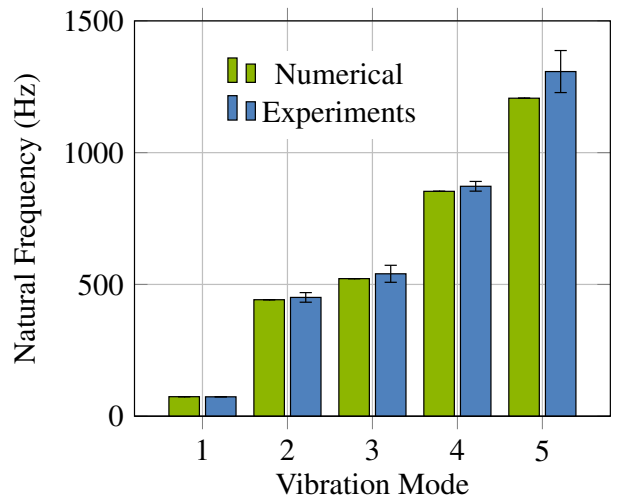

(a) Non delaminated

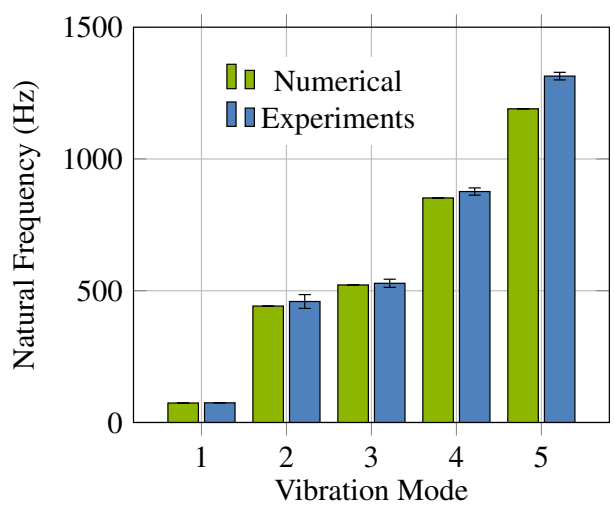

(c) Delamination 2

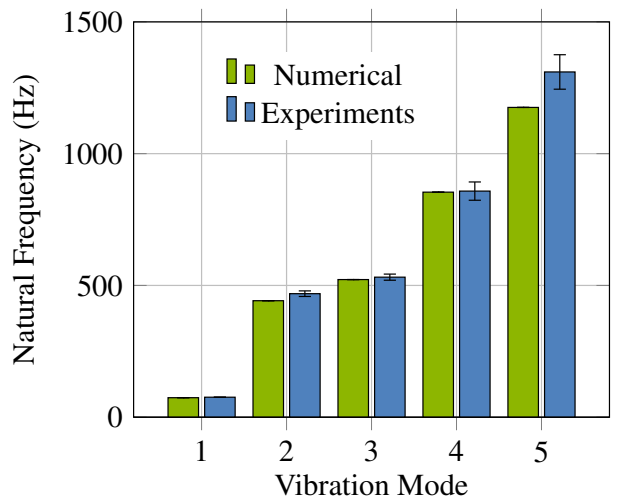

(b) Delamination 1

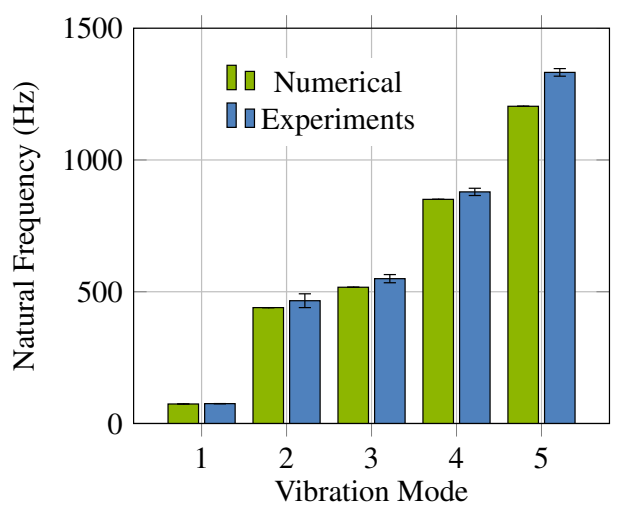

(d) Delamination 3

Figure 3: Numerical and experimental results

\subsection{Application of the delamination diagnosis methodology to the numerically simulated signals}

For the simulated free-decay responses of each specimen, the acceleration is calculated in one node. The simulated signals are $1 s$ long and sampled at 2049 $H z$. Ten realizations for each signal were simulated by adding $20 d B$ white noise to those obtained by transient FEM analysis (see $\$ 3.1$ ). The signals were then subjected to the methodology described in $\$ 2$ with a window length of $W=11$. Figure 4 presents the results: the reconstructed signals using two and four RCs respectively are given in Figure 4(b) and Figure 4(c), the scree diagram in Figure 4(a), and the first two RCs in Figure 4(d).

The percentage of the variance of the decomposition of the signal is shown 


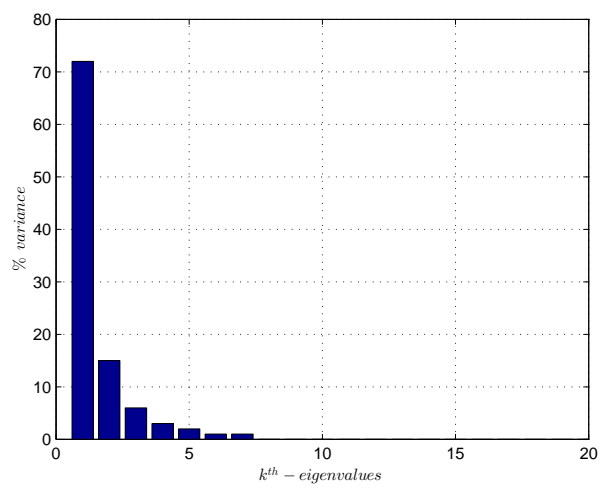

(a) Scree diagram

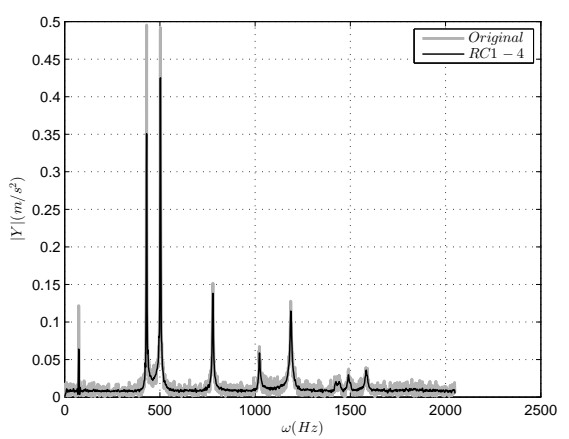

(c) Reconstruction with four RCs

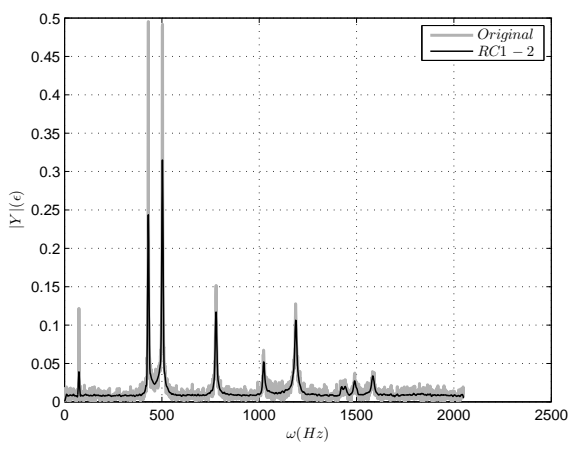

(b) Reconstruction with two RCs

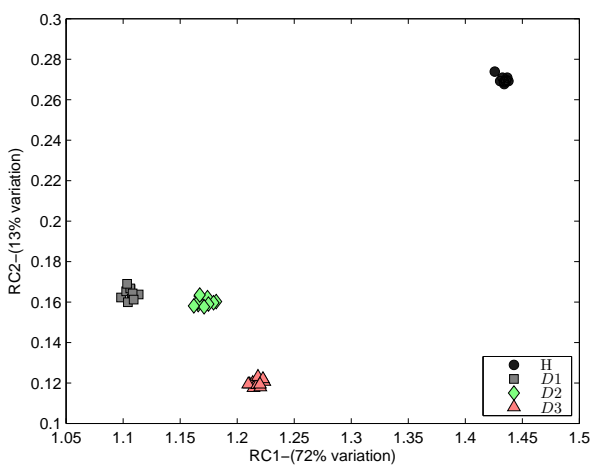

(d) Clustering diagram

Figure 4: Results of the methodology in the numerical analysis.

in Figure 4(a), It can be observed that the first component contains most of the variance (72\%), while the second one has 15 percent, hence the maximum of the variance is contained in the first two components (87\%).

Figure 4(b) and Figure 4(c) present the reconstructed signals by using two and four components respectively. The clustering effect presented by the projection of the original signals onto the two first components is precise enough to identify the different delamination configurations (see Figure 4(d)). The four different cluster groups, corresponding to each delamination scenario, one healthy plate and three delaminated composite plates, are perfectly differentiated. This demonstrates the capabilities of the suggested methodology to 1) detect the delamination in a composite laminate plate and 2) to classify and distinguish between the different damage scenarios for the case of numerically simulated acceleration signals. 


\subsection{Application of the delamination diagnosis methodology to experimentally ex- tracted signals}

The dynamic strain values from the free-decay responses of each specimen were recorded as detailed in \$3.1. Ten signal realizations were obtained for each of the plate scenarios following the experimental process detailed in $\$ 3.2$. The signals were then processed by the methodology presented in $\$ 2$ with a window length of $W=11$ and the results are presented in Figure 5

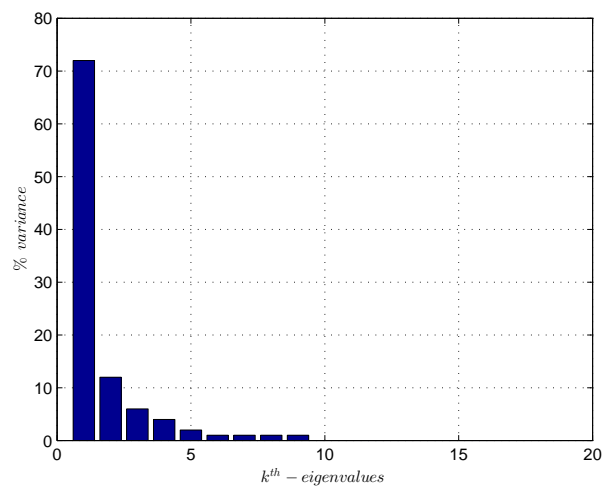

(a) Scree diagram

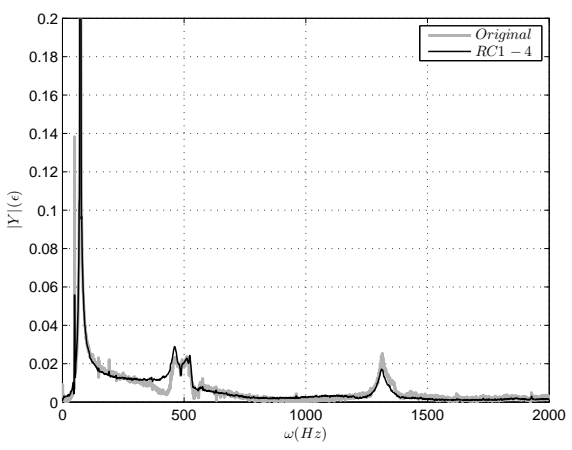

(c) Reconstruction with four RCs

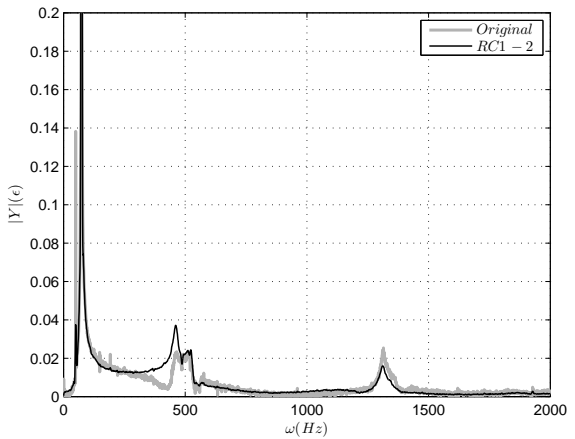

(b) Reconstruction with two RCs

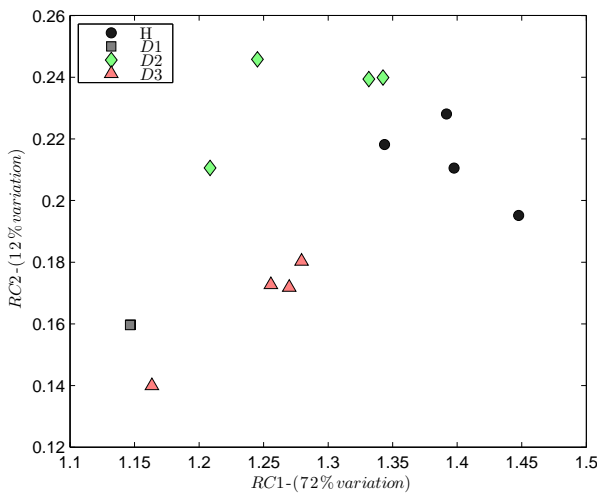

(d) Clustering diagram

Figure 5: Results of the methodolgy in the real composite laminated plates.

The percentage of the variance of the acquired signals is shown in the scree diagrams in Figure 5(a), It can be observed that, similarly to what seen for numerical signals, the first component has the maximum of the variance (still $72 \%$ ), and the second one contains $12 \%$ : the $84 \%$ of the variance is contained in the first two 
components.

Looking at Figure 5(b) and 5(c), it can be seen that by using four RCs the reconstruction signal improves. The authors actually have evidence that by using more components not only the reconstruction improves but also the clustering gets better. Unfortunately more than two components are difficult to visualize and in order to use more components one needs a diagnosis method (in terms of a pattern recognition procedure). The authors are currently working on the development of such a procedure. However, the use of more reconstructed components should be studied in detail because some of our results show that increasing the number of components after a certain number could have an adverse effect on the clusterization. The RCs with smaller content of variance (see Figure 4(a) and Figure 5(a)) contain most of the noise and their consideration is likely to include noise [19].

\subsection{Discussion}

The methodology for delamination detection in composite laminate plates described in \$2 was applied and demonstrated for both numerical case \$3.1 and an experimental benchmark test $(\$ 3.2)$. As defined in the first step of the methodology, the vibratory responses of the healthy categories were first collected and decomposed using a number of PCs. The number of PCs is defined by the number of signals $\mathrm{M}$ and by the window length $W(M x W)$. In the case of FEM analysis the number of PCs into which the signals were decomposed is 110 and in the experimental case it is 44 . In both cases the first two PCs contain most the information (variability) and are very well separated. The percentage of variance contained in the two first PCs is more than 80 percent in both cases with the majority of this contained in the first one.

Figures 4(b) and 5(b) give the reconstruction of the signals using two RCs. It can be observed that the reconstruction for the experimental test is less accurate than for the case of FEM analysis. At low frequencies the reconstruction signal matches closely the original signal. However for higher frequencies $(400 \mathrm{~Hz})$ the reconstruction with two RCs is not very well achieved. This reconstruction can be improved using four RCs as shown in Figures 4(c) and 5(c). This is important to mention because it shows that the developed methodology is able to capture most of the information contained in the original signals and accordingly methods developed based on this methodology should be able to clearly distinguish between different signal categories. The data from three delamination scenarios was projected in the new space made by two RCs. This projection was developed for both cases, as it can be observed in Figure 4(d) and Figure 5(d). The clustering effect 
for the numerical case is perfectly achieved with a 100 percent correct classification of all different delamination scenarios. The distances between points from the same category are small, while the points from different categories are away from each other. The clustering effect, in the case of the experimental analysis is also achieved. Although the classification between categories is clearly achieved, some individual cases could be misclassified. This is because the inter-category distances in this case are comparable to the between-category ones at least for some individual cases. However, as shown in Figure 5(c), the use of more RCs improves the reconstruction of the signal and hence it is expected to improve the clustering effect as well [25]. The above fact opens possibilities for the development of different delamination features and categorization and pattern recognition procedures to improve the currently obtained results.

\section{Conclusions}

This paper presents a purely data driven methodology for delamination detection in composite laminate plates. The methodology based on SSA is able to decompose and reduce the dimension of the vibration response of the dynamics of the composite laminate plates. This methodology is able to detect even small changes in the vibratory response due to the different delamination sizes and scenarios. We have shown that the used reconstruction is able to capture most of the information contained in the originally measured or simulated signals. In this paper also a first attempt for classification according to the different delamination scenarios is made using the first two principal components. But the application of adequate data analysis and pattern recognition procedures should be able to improve the categorization and the clustering effects achieved.

The methodology was applied first to the results from a numerical simulation and secondly to the measured signals from an experimental campaign. The delamination detection, that is the recognition between healthy and delaminated plates, was clearly achieved in both the cases. But as was shown using the first two components provided by the developed methodology it is also possible to distinguish between delamination categories. The delaminated plates were very welldistinguished and separated not only form the healthy scenario but also from the other delamination categories. It is also demonstrated that the use of more RCs is capable to build a much more accurate reconstruction of the original signal.

The delaminated plates were very well distinguished and separated not only from the healthy scenario but also different delamination scenarios were successfully detected. This demonstrates the potential of the methodology for delamination lo- 
calization due to the ability to cluster different delaminated plate scenarios. These results encourage to use the data driven methodology for delamination location and extent in future investigations.

\section{References}

[1] D. Gay, S.V. Hoa, S.W. Tsai SW, Composite Materials: Design and Applications, CRC Press (2003).

[2] G.C. Pardoen, Effect of delamination on the natural frequencies of composite laminates. Journal of composite materials, Journal of composite materials. Vol. 23, No. 12, SAGE (1989), pp. 1200-1215.

[3] G.C. Larsen, P.Berring, D. Tcherniak, P.H. Nielsen, K. Branner, Effect of a Damage to Modal Parameters of a Wind Turbine Blade, Proceedings of EWSHM-7 ${ }^{\text {th }}$ European Workshop on Structural Health Monitoring, 2014.

[4] S.S. Kessler, S.M. Spearing, M.J. Atalla, C.E.S. Cesnik, C. Soutis, Damage detection in composite materials using frequency response methods, Composites Part B: Engineering. Vol. 3, No. 1, Elsevier (2002), pp. 87-95.

[5] S.W. Doebling,C.R. Farrar, M.B. Prime, W.S. Daniel, Damage Identification and Health Monitoring of Structural and Mechanical Systems from Changes in Their Vibration Characteristics: A Literature Review, Los Alamos National Laboratory, Technical report

[6] G.C. Pardoen, J.J. Tracy, Effect of delamination on the natural frequencies of composite laminates, Journal of Composite Materials, Vol. 3, SAGE Publications (1989), pp. 1200-1215.

[7] L.H. Yam, Z. Wei, L. Cheng, W.O. Wong, Numerical analysis of multi-layer composite plates with internal delamination, Computers \& Structures, Vol. 82, No. 7-8, Elsevier (2004), pp. 627-637.

[8] K. Alnefaie, Finite element modeling of composite plates with internal delamination, Composite Structures, Vol. 90, Elsevier (2009), pp. 21-27.

[9] D.X. Lin, R.G. Ni, R.D. Adams, Prediction and measurement of the vibrational damping parameters of carbon glass fiber-reinforced plastics plates, Journal of Composite Material, Vol. 18, SAGE (1984), pp. 132-152. 
[10] P.S. Mujumdar, S. Suryanarayan, Flexural vibrations of beams with delaminations, Journal of Sound and Vibration, Vol. 25, No. 3, Elsevier (1998), pp. 441-461.

[11] H. Abarbanel, Analysis of Observed Chaotic Data, Institute for Nonlinear Science (1996)

[12] H. Krantz, T. Schreiber, Nonlinear time series analysis, Cambridge University Press (1997).

[13] I. Trendafilova, Vibration-based damage detection in structures using time series analysis Proceedings of the Institution of Mechanical Engineers, Part C: Journal of Mechanical Engineering Science, Vol. 220, No. 3, SAGE Publications (2006), pp. 261-272.

[14] J.M. Nichols, W.A. Link, K.D. Murphy, C.C. Olson, A Bayesian approach to identifying structural nonlinearity using free-decay response: application to damage detection in composites, Journal of Sound and Vibration, Vol. 329, No.15, Elsevier (2010), pp.2995-3007.

[15] G. Minak, R. Palazzetti, I. Trendafilova, A. Zucchelli, Localization of a delamination and estimation of its length in a composite laminate beam by the VSHM and pattern recognition methods, Mechanics of Composite Materials, Vol. 46, No. 4, Springer (2010), pp. 387-394.

[16] I. Trendafilova, R. Palazzetti, A. Zucchelli, Delamination Assessment in Structures Made of Composites Based on General Signal Correlation, International Journal of Structural Stability and Dynamics, Vol. 15, No. 1, World Scientific Publishing (2015) 1440022-1-13.

[17] M. Johnson, Waveform based clustering and classification of AE transients in composite laminates using principal component analysis, NDT \& E International, Vol. 35, No. 6, Elsevier (2002), pp. 367-376.

[18] D.A. Tibaduiza, L.E. Mujica, J. Rodellar, Damage classification in structural health monitoring using principal component analysis and selforganizing maps, Structural Control and Health Monitoring, Vol. 20, No. 10, Wiley (2013), pp. 1303-1316.

[19] A.M. Yan, G. Kerschen, P. De Boe, J.C. Golinval, Structural damage diagnosis under varying environmental conditions - Part I: a linear analysis, 
Mechanical Systems and Signal Processing. Vol. 19, No. 4, Elsevier (2005), pp. 847-864.

[20] A.M. Yan, G. Kerschen, P. De Boe, J.C. Golinval, Structural damage diagnosis under varying environmental conditions - Part II: local PCA for non-linear cases, Mechanical Systems and Signal Processing. Vol. 19, No. 4, Elsevier (2005), pp. 865-880.

[21] D. Garcia, I. Trendafilova, A multivariate data analysis approach towards vibration analysis and vibration-based damage assessment. Application for delamination detection in a composite beam, Journal of Sound and Vibrations, Vol. 333, No 25, Elsevier (2014), pp. 7036-7050.

[22] I.T. Jolliffe, Principal Component Analysis, Springer-Verlag New York, 2002.

[23] S.H. Chao, C.H. Loh, Application of singular spectrum analysis to structural monitoring and damage diagnosis of bridges, Structure and Infrastructure Engineering, Vol. 10 No 6, Taylor \& Francis Online (2014), pp. 708-727.

[24] M. Ghil, M.R. Allen, M.D. Dettinger, K. Ide, D. Kondrashov, M.E. Mann, A.W. Robertson, A. Saunders, Y. Tian, F. Varadi, P. Yiou, Advanced spectral methods for climatic time series, Reviews of Geophysics, Vol. 40, No. 1, Wiley (2002), pp. 3-1-3-41.

[25] D. Garcia, I. Trendafilova, H. Al-Bugharbee, Vibration-Based Health Monitoring Approach for Composite Structures Using Multivariate Statistical Analysis, EWSHM-7th European Workshop on Structural Health Monitoring (2014). 